\title{
Comprehensive Health-Related Quality of Life is Influenced by Nocturia and Sleep Disturbance: Investigation Based on the SF-8
}

\author{
SHIGETAKA SUEKANE, KOUSUKE UEDA, SHUNSUKE SUYAMA, TOKUMASA HAYASHI, \\ NORIYUKI TOYOZAWA, MAKI YOSHITAKE, KIYOAKI NISHIHARA, \\ NAO SAKASHITA, NAOHISA UCHIMURA* AND KEI MATSUOKA \\ Department of Urology and *Neuropsychiatry, Kurume University School of Medicine, \\ Kurume 830-0011, Japann
}

Received 20 October 2014, accepted 18 May 2015

J-STAGE advance publication 1 March 2016

\begin{abstract}
Summary: We investigated the influence of nocturia and sleep disturbance on health-related quality of life (HRQOL) using the Medical Outcomes Study 8-item Short Form Health Survey (SF-8) in patients with nocturia. We also assessed the effect of therapeutic intervention by means of an anticholinergic agent on the results of the SF-8. One hundred and eighty-four patients who voided at least once per night were surveyed using the SF-8, Overactive Bladder Symptom Score (OABSS), Pittsburgh Sleep Quality Index (PSQI), and Epworth Sleepiness Scale (ESS). These parameters were also evaluated before and after 12 weeks of imidafenacin treatment in 51 patients with OAB accompanied by nocturia. The SF- 8 physical component summary score (PCS) showed a significant decrease as nighttime voiding frequency increased. The mental health component summary score was 47.1 and 47.6 (which were lower than the standard value of 50) in the group with a nighttime frequency of once and $\geq 3$ /night, respectively. The SF-8 PCS and 6 subscales were negatively associated with nighttime voiding frequency, while the PSQI global score was positively associated with it. Imidafenacin significantly improved the OABSS, PSQI, and ESS, as well as the SF-8 score. This is the first study using the SF-8 to show that nocturia and sleep disturbance have a major influence on comprehensive HRQOL and that the SF- 8 can be used to monitor HRQOL in OAB patients receiving treatment for nocturia.
\end{abstract}

Key words HRQOL, Nocturia, OAB, SF-8, Sleep disturbance

\section{INTRODUCTION}

Nocturia is defined as waking at night one or more times to void [1]. Nocturia markedly impairs both HRQOL and activities of daily living (ADL) [2], with its influence on HRQOL being mediated by disturbance of sleep [3]. Several studies have identified an association among nighttime voiding frequency, the frequency [4] and severity of sleep disturbance, and deterioration of health-related quality of life (HRQOL) [5].

The causes of nocturia are diverse and include
$\mathrm{OAB}$, which was first defined by the International Continence Society in 2002 and is now widely accepted as an established syndrome [1]. Epidemiological research suggests that the number of patients with $\mathrm{OAB}$ in Japan is around 8.1 million, accounting for $12.4 \%$ of the population over 40 years of age [6], and the prevalence of $\mathrm{OAB}$ is expected to continue increasing rapidly with the aging of Japanese society.

Treatment with anticholinergic agents is the firstline therapy for $\mathrm{OAB}$, and many studies have shown that therapeutic intervention with such drugs can improve sleep disorders [7] and HRQOL [8] along with

Corresponding author: Shigetaka Suekane, M.D., Ph.D., Department of Urology, Kurume University School of Medicine, 67 Asahi-machi, Kurume 8300011, Japan. Tel: +81-942-31-7572 Fax: +81-942-34-2605 Email: suekane@med.kurume-u.ac.jp

Abbreviations: ADL, activities of daily living; BP, bodily pain; BPH, benign prostatic hyperplasia; EPIC, Expanded Prostate Cancer Index Composite; ESS, Epworth Sleepiness Scale; GH, general health; HRQOL, health-related quality of life; MCS, mental health component summary score, MH, mental health; OABSS, Overactive Bladder Symptom Score; PF, physical functioning; PCS, physical health component summary score; PSQI, Pittsburgh Sleep Quality Index; RE, role emotional; RP, Role Physical; SF, social functioning; SF-8, The Medical Outcomes Study 8-item Short Form Health Survey; VT, vitality. 
nocturia in $\mathrm{OAB}$ patients.

HRQOL questionnaires can be classified as comprehensive or disease-specific. The SF-36 is a typical comprehensive questionnaire and it is used worldwide. The Medical Outcomes Study 8-item Short Form Health Survey (SF-8) is a short version of SF-36 that permits comparison of HRQOL between patients with various diseases or comparison of health between patients and the general population [9]. Compared with the SF-36, the SF-8 is more practical for evaluation of the actual condition of patients and their clinical response, in combination with other questionnaires, because of its simplicity. A prostate cancer-specific questionnaire, the Expanded Prostate Cancer Index Composite (EPIC), is recommended for use with comprehensive questionnaires. The SF-12, another short version of the SF-36, has often been employed with EPIC in overseas studies, while use of EPIC with the SF-8 has been verified in Japan [10]. For evaluating the influence of nocturia, however, the SF-8 has never been used by itself or in combination with other questionnaires.

In this study, we investigated the influence of nocturia and sleep disturbance on HRQOL using the SF-8, PSQI, and ESS in patients with nocturia, which was defined as one or more episodes of voiding per night (Study 1). We also assessed these parameters in patients who had nocturia associated with $\mathrm{OAB}$ before and after 12 weeks of treatment with an anticholinergic agent, imidafenacin, (Study 2).

\section{METHODS}

Influence of nocturia and sleep disturbance on HRQOL (Study 1)
This study was performed at the Departments of Urology and Psychiatry of Kurume University and related hospitals in Japan in accordance with the Helsinki Declaration after approval of the Kurume University Ethics Committee. During the period between March 2009 and November 2011, patients with nocturia (at least one voiding episode per night) were enrolled after they gave informed consent (Fig. 1).

One hundred and eighty-four patients were surveyed using a questionnaire prepared for this study that included the OABSS [11], SF-8 [9], Pittsburgh Sleep Quality Index (PSQI) [12], and Epworth Sleepiness Scale (ESS) [13].

HRQOL was evaluated by the SF-8, which includes 8 subscales ('physical functioning (PF)', 'role physical (RP)', 'bodily pain (BP)', 'general health (GH)', 'vitality (VT)', 'social functioning (SF)', 'role emotional (RE)', and 'mental health (MH)'), and 2 summary scores ("physical health component summary score (PCS)' and 'mental health component summary score (MCS)') that are calculated from the 8 subscale scores. In this study, the score for each subscale, the PCS, and the MCS were calculated using a normbased scoring method based on the national standard values determined from large-scale survey data for the general Japanese population (the mean score and standard deviation of the general Japanese population are 50 and 10, respectively) [9].

For evaluation of sleep disturbance, the global and component scores of the PSQI and the total score of the ESS were investigated. Cut-off values of 5.5 for the PSQI [14] and 11 for the ESS [13] have been established as defining the presence/absence of sleep disturbance.

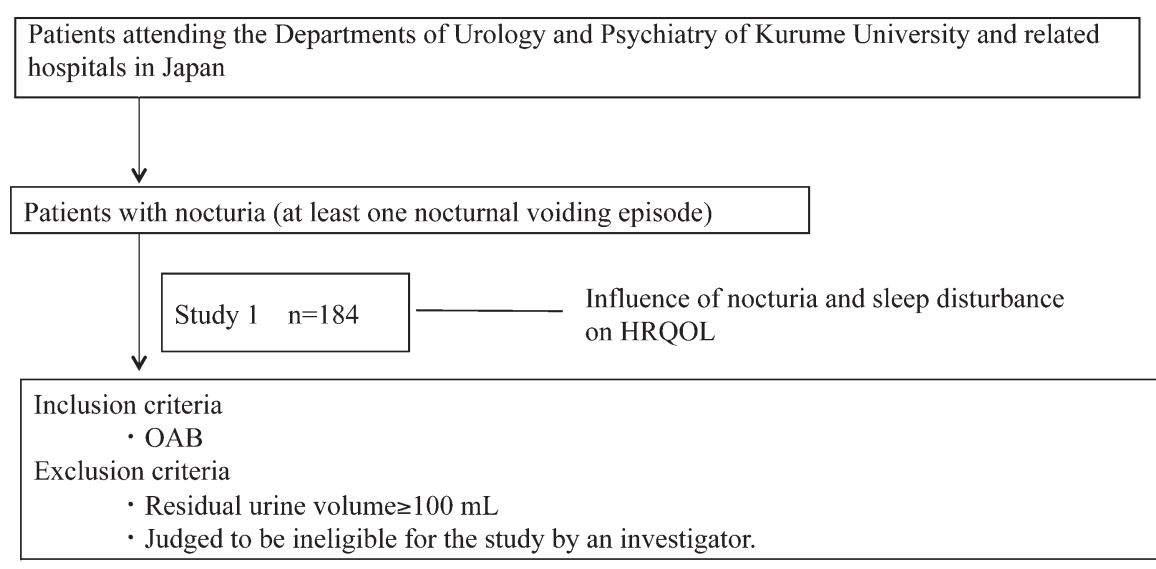

Study $2 \mathrm{n}=51 \quad$ Evaluation of the response to imidafenacin

Fig. 1. Flow chart of patient disposition 
HRQOL in $O A B$ patients with nocturia treated with an anticholinergic agent (Study 2)

Study 2 was performed in 51 patients with $\mathrm{OAB}$ who met the inclusion criteria for Study 1 and did not fit either of the exclusion criteria (Fig. 1), which were a residual urine volume $\geq 100 \mathrm{~mL}$ and ineligibility for the study based on the judgment of the investigator.

Imidafenacin was administered at a dose of 0.1$0.2 \mathrm{mg}$ twice daily (after breakfast and dinner) for 12 weeks. The effect of the drug was investigated at baseline and every 4 weeks during the treatment period using the OABSS, SF-8, PSQI, and ESS.

For statistical analysis, the Jonckheere-Terpstra trend test was used to evaluate the association between an increase of the OABSS nighttime voiding frequency score and parameters such as the SF-8, PSQI, and ESS scores. The Cochrane-Armitage trend test was employed to estimate the change in the percentage of patients with a PSQI global score or ESS total score higher than each cut-off value. The Wilcoxon signed-rank test or McNemar test was applied to compare the parameters at each time of assessment during treatment with those at baseline. In all analyses, $\mathrm{p}<0.05$ was considered to indicate significance.

\section{RESULTS}

Influence of nocturia and sleep disturbance on HRQOL (Study 1)
The patient characteristics are summarized in Table 1. There were 184 patients (132 men and 52 women) with a mean age of $70.9 \pm 10.3$ years. The most common underlying disease was $\mathrm{OAB}$, which was diagnosed in 94 patients $(51.1 \%)$. Among the male patients, $67(50.8 \%)$ had benign prostatic hyperplasia (BPH). Other associated diseases included hypertension $(22.3 \%)$ and diabetes $(8.2 \%)$.

SF-8 scores stratified by the OABSS nighttime voiding frequency score are shown in Fig. 2. The PCS showed a significant decrease as nocturia frequency increased. The MCS values were 47.1 and 47.6 (which were lower than the standard value of 50) in the groups with a nighttime voiding frequency score of 1 (once/ night group) and a score of 3 ( $\geq 3 /$ night group), respectively. There was a negative association with an increasing frequency of nocturia for the PCS and 6 subscales of the SF-8 (PF, RP, GH, VT, SF, and MH).

The PSQI and ESS scores for nocturia are summarized in Table 2. As nocturia increased, the PSQI global score showed a significant increase. In the $\geq 3$ / night group, the mean score was 7.28, which exceeded the cut-off value of 5.5. Similarly, the PSQI component scores for 'sleep quality', 'sleep efficiency', and 'sleep disturbance' showed an increase, indicating that these items were significantly associated with the nighttime voiding frequency score. The percentage of patients with a PSQI global score $\geq 5.5$ also increased and the association was significant. The ESS total score rose slightly with an increase of nocturia, but the

TABLE 1

Patients characteristics

\begin{tabular}{llc}
\hline No. of patients & & 184 \\
\hline Age (Mean \pm SD) & & $70.9 \pm 10.3$ \\
\hline Gender & Male / female & $132 / 52$ \\
\hline Comorbidity & Diabetes & $15(8.2 \%)$ \\
& Hypertension & $41(22.3 \%)$ \\
& Cerebrovascular disease & $12(6.5 \%)$ \\
& Benign prostatic hyperplasia & $67(50.8 \%$, male) \\
& Prostatitis & $3(2.3 \%$, male $)$ \\
& Prostate cancer & $4(3.0 \%$, male $)$ \\
& Overactive bladder & $94(51.1 \%)$ \\
& Others & $32-$ \\
\hline Concomitant drug & $\alpha$-receptor blocking drug & $61(46.2 \%$, male $)$ \\
& Antidepressant & $2(1.1 \%)$ \\
& Anti-anxiety agent/sleeping drug & $44(23.9 \%)$ \\
& Others & $23(12.5 \%)$
\end{tabular}




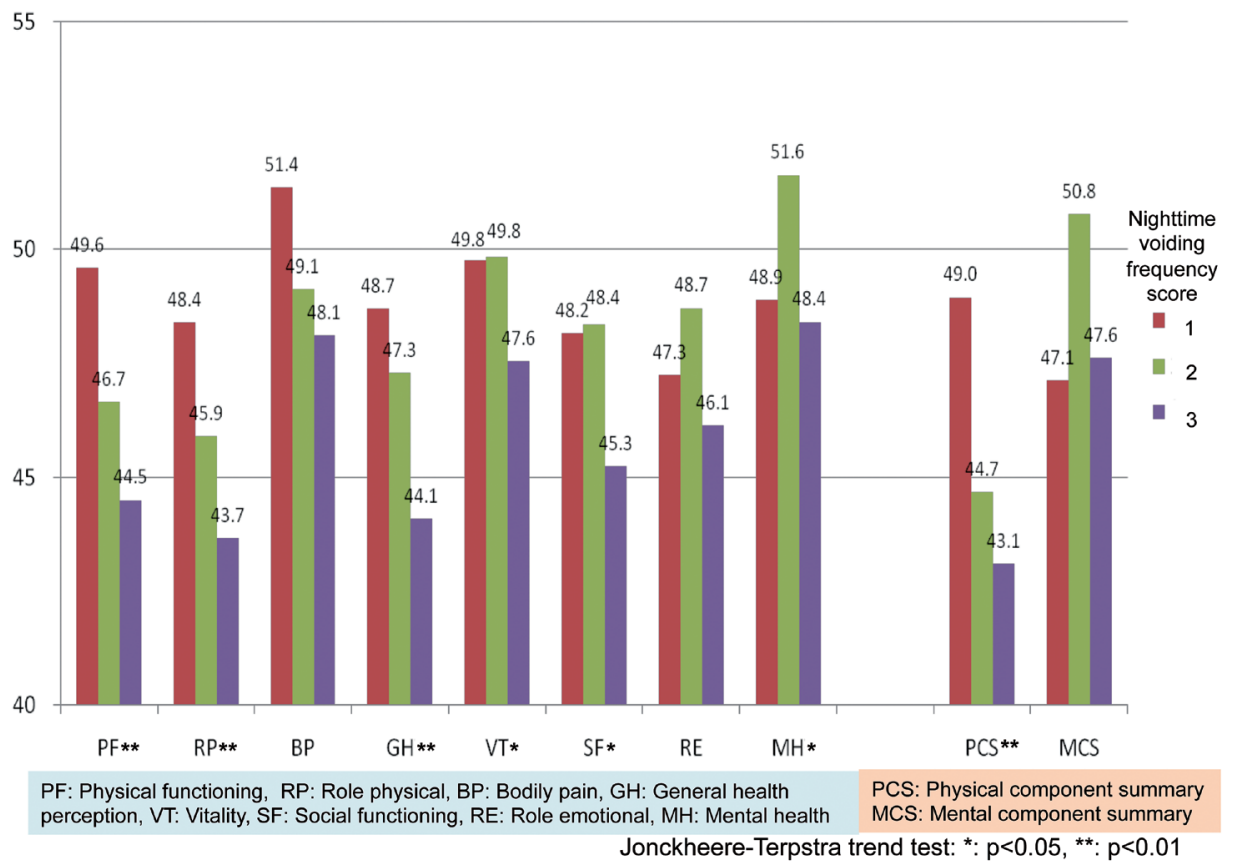

Fig. 2. SF-8 scores stratified by the OABSS nighttime voiding frequency score.

TABLE 2.

Comparison of PSQI and ESS scores stratified according to the OABSS nighttime voiding frequency score

\begin{tabular}{|c|c|c|c|c|c|}
\hline \multicolumn{2}{|c|}{$\begin{array}{l}\text { Nighttime voiding frequency score in OABSS } \\
\text { (Voiding frequency/ night) }\end{array}$} & $\begin{array}{c}1 \\
(1)\end{array}$ & $\begin{array}{l}2 \\
(2)\end{array}$ & $\begin{array}{c}3 \\
(\geq 3)\end{array}$ & \multirow[t]{2}{*}{$\begin{array}{c}\text { P-value } \\
\text { trend }\end{array}$} \\
\hline No. pts & & 16 & 56 & 112 & \\
\hline \multirow[t]{9}{*}{ PSQI } & Sleep quality & $1.06 \pm 0.57$ & $1.14 \pm 0.72$ & $1.46 \pm 0.83$ & 0.0032 \\
\hline & Sleep latency & $0.75 \pm 0.86$ & $1.11 \pm 0.91$ & $1.23 \pm 1.00$ & 0.1110 \\
\hline & Sleep duration & $0.88 \pm 0.81$ & $0.96 \pm 0.87$ & $1.09 \pm 1.00$ & 0.3713 \\
\hline & Sleep efficiency & $0.13 \pm 0.34$ & $0.21 \pm 0.49$ & $0.90 \pm 1.15$ & $<0.0001$ \\
\hline & Sleep disturbance & $0.56 \pm 0.51$ & $0.88 \pm 0.54$ & $1.19 \pm 0.66$ & $<0.0001$ \\
\hline & Hypnotic medication use & $0.19 \pm 0.75$ & $0.55 \pm 1.11$ & $0.71 \pm 1.22$ & 0.1521 \\
\hline & Daytime dysfunction & $0.44 \pm 0.63$ & $0.55 \pm 0.69$ & $0.70 \pm 0.76$ & 0.1168 \\
\hline & Global score & $4.00 \pm 2.71$ & $5.41 \pm 3.13$ & $7.28 \pm 4.36$ & 0.0004 \\
\hline & $\%$ of pts with $\mathrm{PSQI}>5.5$ & $31.3 \%$ & $50.0 \%$ & $61.6 \%$ & 0.0165 \\
\hline \multirow[t]{2}{*}{ ESS } & Total score & $4.13 \pm 2.45$ & $4.77 \pm 3.65$ & $5.10 \pm 2.95$ & 0.0918 \\
\hline & $\%$ of pts with ESS $>11$ & $0.0 \%$ & $7.1 \%$ & $5.4 \%$ & 0.8099 \\
\hline
\end{tabular}

Data are expressed as the mean \pm SD, unless otherwise indicated. Statistical analysis was performed by using the Jonckheere-Terpstra trend test to evaluate the changes of PSQI and ESS scores, or with the Cochrane-Armitage trend test to evaluate the change in the percentage of patients with sleep disturbance.

change was not significant.

\section{$H R Q O L$ in $O A B$ patients with nocturia receiving treat-} ment of an anticholinergic agent (Study 2)

The patient characteristics are listed in Table 3. There were 51 patients ( 21 men and 30 women) with a mean age of $72.6 \pm 9.3$ years. Thirteen male patients had BPH (61.9\%). Other associated diseases were hypertension $(37.3 \%)$, diabetes $(13.7 \%)$, and cerebrovascular disease $(9.8 \%)$.

Serial changes of the OABSS are summarized in Table 4. Compared with the baseline value $(2.61 \pm 0.67)$, 
TABLE 3.

Patients characteristics treated by imidafenacin

\begin{tabular}{llc}
\hline No. of patients & \multicolumn{2}{c}{51} \\
\hline Age (Mean \pm SD) & \multicolumn{2}{c}{$72.6 \pm 9.3$} \\
\hline Gender & Male / female & $21 / 30$ \\
\hline Comorbidity & Diabetes & $7(13.7 \%)$ \\
& Hypertension & $19(37.3 \%)$ \\
& Cerebrovascular disease & $5(9.8 \%)$ \\
& Benign prostatic hyperplasia & $13(61.9 \%$, male $)$ \\
& Prostatitis & $1(4.8 \%$, male $)$ \\
& Others & 17 \\
\hline \multirow{2}{*}{ Concomitant therapy } & Behavioral therapy & $3(5.9 \%)$ \\
& $\alpha$-receptor blocking drug & $13(61.9 \%$, male $)$ \\
& Anti-anxiety agent/sleeping drug & $13(25.5)$ \\
& Others & $2(3.9)$ \\
\hline
\end{tabular}

TABLE 4.

Changes of $O A B S S$ in $O A B$ patients with nocturia treated by imidafenacin

\begin{tabular}{llcccc}
\hline & baseline & $4 \mathrm{w}$ & $8 \mathrm{w}$ & $12 \mathrm{w}$ \\
\hline OABSS (N=51) & Daytime frequency score & $1.06 \pm 0.42$ & $0.78 \pm 0.46^{* *}$ & $0.84 \pm 0.42^{* *}$ & $0.67 \pm 0.52^{* * *}$ \\
& Nighttime frequency score & $2.61 \pm 0.67$ & $2.25 \pm 0.84^{* * *}$ & $2.22 \pm 0.92^{* * *}$ & $2.12 \pm 1.01^{* * *}$ \\
& Urgency score & $3.47 \pm 0.97$ & $1.73 \pm 1.46^{* * *}$ & $1.61 \pm 1.47^{* * *}$ & $1.53 \pm 1.33^{* * *}$ \\
& Urgency incontinence score & $1.92 \pm 1.78$ & $1.06 \pm 1.41^{* * *}$ & $1.06 \pm 1.41^{* * *}$ & $1.04 \pm 1.39^{* * *}$ \\
& Total OABSS & $9.06 \pm 2.57$ & $5.82 \pm 2.89^{* * *}$ & $5.73 \pm 3.06^{* * *}$ & $5.35 \pm 3.06^{* * *}$ \\
\hline
\end{tabular}

Data are expressed as mean $\pm \mathrm{SD}$. Statistical analysis was performed using Wilcoxon signed-rank test. $* * \mathrm{p}<0.01$; $* * * \mathrm{p}<0.001$.

treatment with imidafenacin significantly reduced the nighttime voiding frequency score at 4,8 , and 12 weeks $(2.25 \pm 0.84(\mathrm{p}<0.001), 2.22 \pm 0.92(\mathrm{p}<0.001)$, and $2.12 \pm 1.01(\mathrm{p}<0.001)$, respectively).

Serial changes of the SF-8 score are summarized in Fig. 3. The PCS was 39.5 before starting imidafenacin administration, and it increased to 42.3 $(\mathrm{p}=0.0713), 43.6(\mathrm{p}<0.05)$, and $42.5(\mathrm{p}=0.0618)$ after 4,8 , and 12 weeks of administration, respectively, with a significant difference between the baseline and 8 -week scores. The MCS was 47.5 before administration and it increased significantly to $49.7(\mathrm{p}<0.05)$ and $50.1(\mathrm{p}<0.05)$ after 8 and 12 weeks, respectively. Regarding the subscales, significant improvement compared with baseline was found for RP at 8 weeks, $\mathrm{BP}$ and VT at 8, 12 weeks, $\mathrm{MH}$ at 4 and 8 weeks, and $\mathrm{GH}$ and RE at 4, 8, and 12 weeks.

Serial changes of the PSQI and ESS are summarized in Table 5. The PSQI global score was $6.51 \pm 4.10$ at baseline, whereas the score decreased to $5.42 \pm 3.33$ $(\mathrm{p}=0.0503), \quad 5.09 \pm 3.10 \quad(\mathrm{p}<0.01)$, and $5.44 \pm 3.22$ $(\mathrm{p}=0.0793)$ at 4,8 , and 12 weeks, respectively, all of which were below the cut-off value of 5.5. In addition, the percentage of patients with a score $>5.5$ was $55.8 \%$ at baseline, but decreased to $37.2 \%(\mathrm{p}<0.05), 37.2 \%$ $(\mathrm{p}<0.05)$, and $46.5 \%$ at 4,8 , and 12 weeks, respectively. The PSQI component scores for 'sleep quality' and 'sleep latency' were also significantly improved after 4, 8, and 12 weeks compared with those at baseline. Moreover, 'daytime dysfunction' showed significant improvement at 8 weeks. The ESS total score was also significantly improved at 4,8 , and 12 weeks compared with the baseline value. At baseline, $7.1 \%$ of patients had a score higher than the cut-off value of 11 and this percentage decreased to $2.4 \%$ after 4 weeks.

Regarding adverse events caused by imidafenacin therapy, anticholinergic adverse effects (dry mouth and constipation) were occasionally noted, but none of these became serious. 


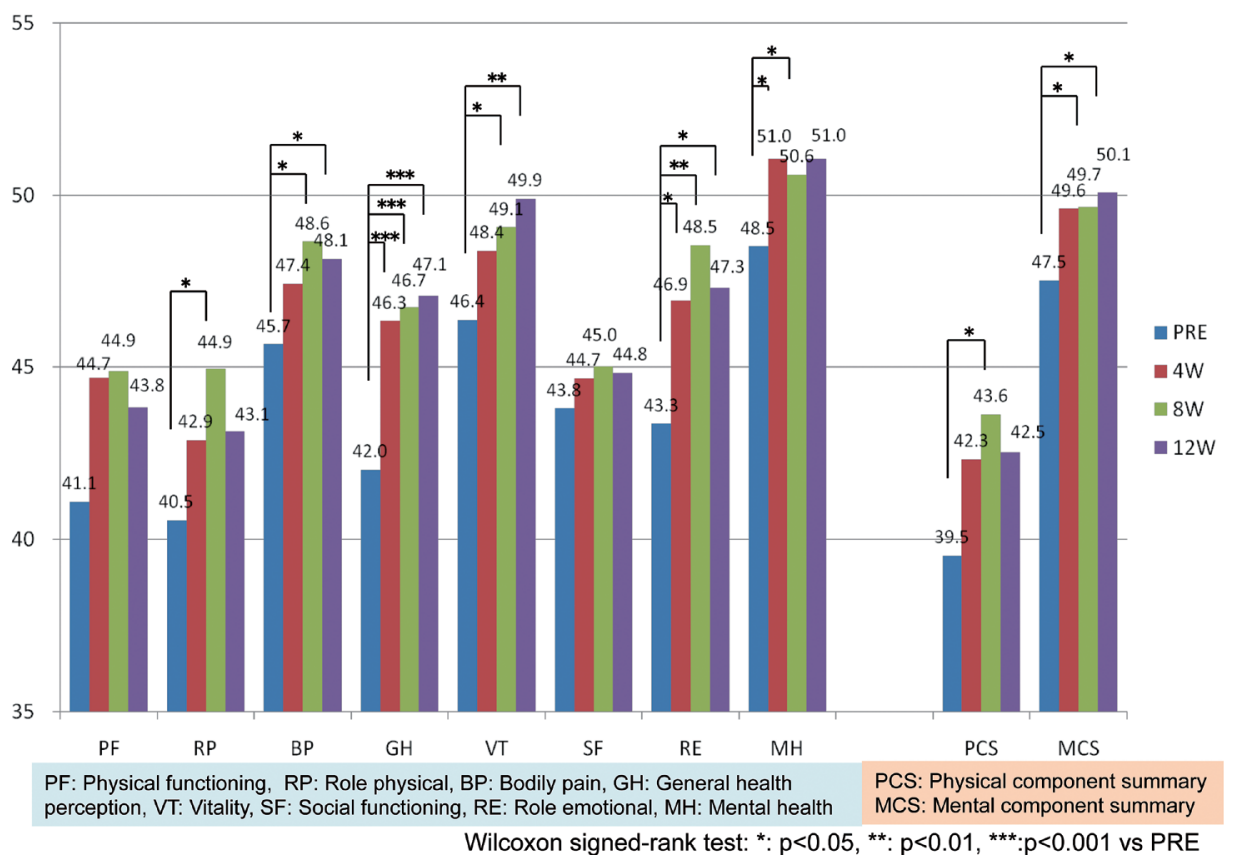

Fig. 3. Changes in SF-8 scores in OAB patients with nocturia treated with imidafenacin.

TABLE 5 .

Changes of the PSQI and ESS scores in OAB patients with nocturia receiving imidafenacin

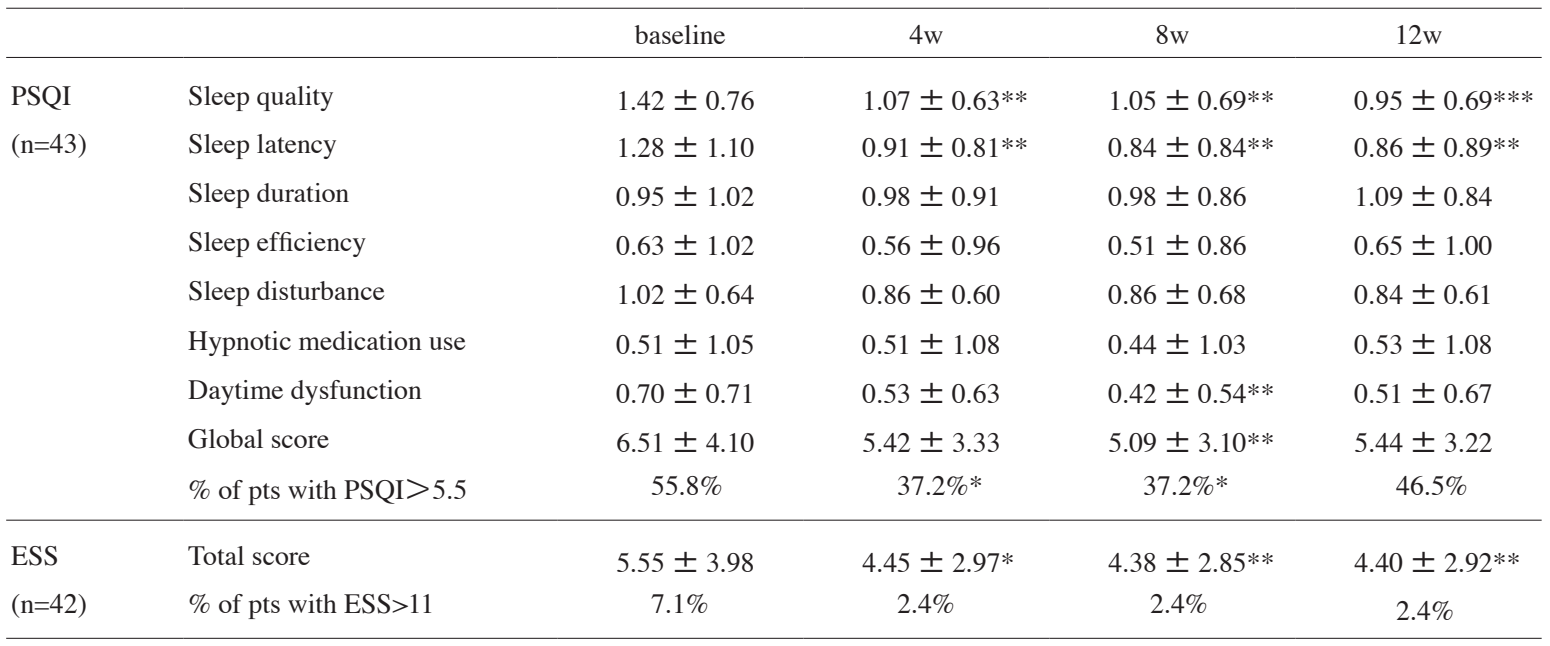

Data are expressed as the mean $\pm \mathrm{SD}$, unless otherwise indicated. Statistical analysis was performed by using the Wilcoxon signedrank test to evaluate the changes of PSQI and ESS scores, or with McNemar's test to evaluate the change in the percentage of patients with sleep disturbance. $* \mathrm{p}<0.05 ; * * \mathrm{p}<0.01 ; * * \mathrm{p}<0.001$.

\section{DISCUSSION}

While a negative influence of nocturia on HRQOL has been reported by many authors, few studies have evaluated the influence of nocturia and sleep disturbance on comprehensive HRQOL using the SF-8. This is the first study in which the SF-8 was employed to demonstrate that an increase of nighttime voiding fre- quency has a marked influence on not only sleep disturbance but also HRQOL in Japanese patients with nocturia. Since the SF-8 is a comprehensive QOL questionnaire, our findings indicate that nocturia influences both sleep and overall ADL.

A previous Dutch study using RAND-36[3] showed that subjects who found nocturia to be bothersome were mentally and physically less healthy than those 
without nocturia. A Swedish study [15] using an original questionnaire found that an increase of nocturia was associated with the impairment of both physical and mental health. Our findings support these reports as we showed that an increase in nighttime voiding frequency was associated with lower scores for the PCS and mental subscales (SF and MH) of the SF-8. Thus, an increase in nighttime voiding has a negative influence on both mental and physical health to some extent, showing that nocturia is not just a nocturnal problem.

The SF-8 allows us to compare HRQOL between patients with different diseases and also to make international comparisons of diseases. In this study, patients who voided twice/night had a PCS of 44.7 and an MCS of 50.8, while those voiding $\geq 3$ times/night had a PCS of 43.1 and an MCS of 47.6. These values are similar to or lower than those obtained in patients with diabetes (47.20 and 48.22, respectively), hypertension (48.33 and 49.32, respectively), and osteoarthritis (45.73 and 49.16, respectively) in a study performed in the US [16], or those for outpatients with cancer (44.3 and 47.6, respectively) obtained in Japan [17]. It is noteworthy that our patients voiding $\geq 3$ times/night had a very low PCS score, which was similar to that of cancer patients. This indicates that nocturia can seriously impair HRQOL and requires therapeutic intervention.

The results of our Study 1 showed that an increase of nighttime voiding frequency is associated with a deterioration of 'Sleep quality', rather than a decrease of 'Sleeping duration'. This is noteworthy since it suggests that a qualitative, rather than a quantitative factor, contributes to sleep disturbance caused by an increase of voiding. Waking up during the night to void impairs the continuation of sleep due to the influence of intermittent awakening. Thus, voiding causes difficulty in returning to sleep due to factors such as behavioral arousal, the effect of the light in the toilet, or stimulation by the low room temperature in winter. All of these factors can shorten the sleeping time and lead to lighter sleep. Intermittent awakening suppresses slow-wave sleep that is required for true recuperation of physiological function. Shortening of the sleeping time also negatively influences metabolic and endocrine functions [18]. Moreover, insufficient slow-wave sleep impairs the regulation of various biological hormones, even if the total sleeping time is maintained [19], preventing recovery from fatigue and resting of the brain. Thus, nocturia-induced intermittent awakening may qualitatively and quantitatively impair sleep, as described above, and the present findings suggest that one mechanism underlying the negative influence of increased nighttime voiding on HRQOL is dysregulation of biological functions due to impaired sleep, particularly reduced sleep quality.

$\mathrm{OAB}$ is a cause of nocturia that is mainly treated by using anticholinergic agents. Intervention with such drugs may be useful for reducing nighttime voiding and several anticholinergic agents are currently available in Japan. Imidafenacin was released in Japan in 2007 [20,21]. It is more bladder-selective compared with other drugs [22], leading to mild anticholinergic adverse effects such as dry mouth and constipation [23], along with good long-term (52 weeks) efficacy, safety, and tolerability $[24,25]$ in OAB patients. Because of these characteristics, imidafenacin is widely employed for the treatment of $\mathrm{OAB}$ in elderly Japanese patients. Use of imidafenacin to treat OAB accompanied by nocturia has also attracted attention, and the improvement of nocturia, sleep disturbance, and HRQOL have been reported $[7,8]$. The effect of imidafenacin on nocturia with $\mathrm{OAB}$ has been established, so we monitored the change of SF-8 score after use of this anticholinergic agent as a medical intervention.

Treatment with imidafenacin significantly improved nocturia in the present study. Sleep disturbance was also improved according to the PSQI and ESS. Moreover, HRQOL was improved according to the SF-8 (PCS and MCS), which revealed either significant improvement or a tendency for improvement from 8 weeks. Similar findings have recently been reported by Nagaoka et al. [26] and Wada et al. [27], who concluded that imidafenacin can be useful for the treatment of nocturia, sleep disturbance, and HRQOL.

In general, comprehensive scales are considered to be less appropriate for assessing the effect of medical intervention on specific diseases compared with disease-specific scales. However, a therapeutic effect of imidafenacin was detected in the present study by the SF-8, a comprehensive scale, which suggests that the SF-8 can be used to monitor HRQOL in OAB patients with nocturia receiving treatment with anticholinergics.

\section{CONCLUSION}

This is the first study using the SF-8 to show that nocturia and sleep disturbance have a major influence on comprehensive HRQOL, similar to that of other chronic disease such as diabetes, hypertension, and even cancer in outpatients, and that the SF- 8 can be used to monitor HRQOL in $\mathrm{OAB}$ patients receiving treatment for nocturia. 


\section{REFERENCES}

1. Abrams P, Cardozo L, Fall M, Griffiths D, Rosier P et al. The standardisation of terminology of lower urinary tract function: report from the Standardisation Sub-committee of the International Continence Society. Neurourol Urodyn 2002; 21:167-178.

2. Donovan JL. Measuring the impact of nocturia on quality of life. BJU Int 1999; 84 Suppl 1:21-25.

3. van Dijk L, Kooij DG, Schellevis FG, Kaptein AA, Boon TA et al. Nocturia: impact on quality of life in a Dutch adult population. BJU Int 2004; 93:1001-1004.

4. Asplund R, and Aberg H. Nocturnal micturition, sleep and well-being in women of ages 40-64 years. Maturitas 1996; 24:73-81.

5. Coyne KS, Zhou Z, Bhattacharyya SK, Thompson CL, Dhawan $\mathrm{R}$ et al. The prevalence of nocturia and its effect on health-related quality of life and sleep in a community sample in the USA. BJU Int 2003; 92:948-954.

6. Homma Y, Yamaguchi O, and Hayashi K. An epidemiological survey of overactive bladder symptoms in Japan. BJU Int 2005; 96:1314-1318.

7. Takeda M, Takahashi S, Nishizawa O, Gotoh M, and .Yoshida M. Imidafenacin, a novel anticholinergic, significantly improves both nocturia and sleep disorders in $\mathrm{OAB}$ patients. - EPOCH (Evaluation of anticholinergics in patients with overactive bladder and nocturia for caredhealth) study -. Jpn J Urol Surg 2009; 22:53-60. (in Japanese)

8. Takeda M, Takahashi S, Nishizawa O, Gotoh M, Yoshida $\mathrm{M}$ et al. Imidafenacin, a novel anticholinergic, significantly improves nocturia, sleep disorders and quality of life in OAB patients. - EVOLUTION (Evaluating the value of nocturia-quality of life questionnaire utilization with treatment of imidafenacin for OAB patients suffering from nocturia) study-. Jpn J Urol Surg 2010; 23:1443-1452. (in Japanese)

9. Fukuhara S, and Suzukamo Y. Manual of the SF-8 Japanese Version. Institite for Health Outcome and Process Evaluation Research, Kyoto, 2004.

10. Sugimoto M, Takegami M, Suzukamo Y, Fukuhara S, and Kakehi Y. Health-related quality of life in Japanese men with localized prostate cancer: assessment with the SF-8. Int J Urol 2008; 15:524-528.

11. Homma Y, Yoshida M, Seki N, Yokoyama O, Kakizaki H et al. Symptom assessment tool for overactive bladder syndrome-overactive bladder symptom score. Urology 2006; 68:318-23.

12. Buysse DJ, Reynolds CF 3rd, Monk TH, Berman SR, and Kupfer DJ. The Pittsburgh Sleep Quality Index: a new instrument for psychiatric practice and research. Psychiatry Res 1989; 28:193-213.

13. Johns MW. A new method for measuring daytime sleepiness: the Epworth sleepiness scale. Sleep 1991; 14:540-545.

14. Doi Y, Minowa M, Uchiyama M, Okawa M, Kim K et al. Psychometric assessment of subjective sleep quality using the Japanese version of the Pittsburgh Sleep Quality Index (PSQI-J) in psychiatric disordered and control subjects.
Psychiatry Res 2000; 97:165-172.

15. Asplund R, Marnetoft SU, Selander J, and Akerström B. Nocturia in relation to somatic health, mental health and pain in adult men and women. BJU Int 2005; 95:816-819.

16. Turner-Bowker DM, Bayliss MS, Ware JE Jr, and Kosinski M. Usefulness of the SF-8 Health Survey for comparing the impact of migraine and other conditions. Qual Life Res 2003; 12:1003-1012.

17. Uramoto H, Kagami S, Iwashige A, and Tsukada J. Evaluation of the quality of life between inpatients and outpatients receiving cancer chemotherapy in Japan. Anticancer Res 2007; 27:1127-1132.

18. Spiegel K, Leproult R, and Van Cauter E. Impact of sleep debt on metabolic and endocrine function. Lancet 1999; 354:1435-1439.

19. Tasali E, Leproult R, Ehrmann DA, and Van Cauter E. Slow-wave sleep and the risk of type 2 diabetes in humans. Proc Natl Acad Sci USA 2008; 105:1044-1049.

20. Leone Roberti Maggiore U, Scala C, Venturini PL, and Ferrero S. Imidafenacin for the treatment of overactive bladder. Expert Opin Pharmacother. Published online: 6 May 2013; DOI:10.1517/14656566.2013.796930)

21. Masumori N. Long-term safety, efficacy, and tolerability of imidafenacin in the treatment of overactive bladder: a review of the Japanese literature. Patient Prefer Adherence 2013; 7:111-20.

22. Kobayashi F, Yageta Y, Yamazaki T, Wakabayashi E, Inoue $\mathrm{M}$ et al. Pharmacological effects of imidafenacin (KRP197/ONO-8025), a new bladder selective anti-cholinergic agent, in rats. Comparison of effects on urinary bladder capacity and contraction, salivary secretion and performance in the Morris water maze task. Arzneimittelforschung 2007; 57:147-154.

23. Homma Y, and Yamaguchi O. Imidafenacin Study Group: A randomized, double-blind, placebo- and propiverine-controlled trial of the novel antimuscarinic agent imidafenacin in Japanese patients with overactive bladder. Int J Urol 2009; 16:499-506.

24. Homma Y, and Yamaguchi O. Long-term safety, tolerability, and efficacy of the novel anti-muscarinic agent imidafenacin in Japanese patients with overactive bladder. Int J Urol 2008; 15:986-991.

25. Zaitsu M, Mikami K, Ishida N, and Takeuchi $T$. Comparative Evaluation of the Safety and Efficacy of Long-Term Use of Imidafenacin and Solifenacin in Patients with Overactive Bladder: A Prospective, Open, Randomized, Parallel-Group Trial (the LIST Study). Adv Urol 2011; 2011:1-10.

26. Nagaoka A, Sakurai T, Naito S, Nishida H, Kawazoe H et al. Sleep disorders and HRQOL were significantly improved by imidafenacin, an anticholinergic agent, in $\mathrm{OAB}$ patients with nocturia. Jpn J Urol Surg 2011; 24:1649-1656. (in Japanese)

27. Wada N, Watanabe M, Kita M, Matsumoto S, Osanai H et al. Effect of imidafenacin on nocturia and sleep disorder in patients with overactive bladder. Urol Int 2012; 89:215-221. 